\title{
Shared Resource Network-Aware Impact Determination Algorithms for Service Workflow Deployment
}

\author{
Hendrik Moens ${ }^{\mathrm{a}}$, Filip De Turck ${ }^{\mathrm{a}}$ \\ ${ }^{a}$ Ghent University - iMinds, Department of Information Technology, \\ Gaston Crommenlaan 8/201, B-9050 Gent, Belgium
}

\begin{abstract}
Some service providers offer services for which physical devices, terminals and servers are installed at the customer's site, e.g. in a hospital setting where physical terminals are installed in patient rooms and used for communication. These devices work together to provide the service, forming service workflows. Due to a recent trend where parts of the management system for such services is offloaded to cloud environments, such services can no longer be isolated in a private subnet, that is specifically dimensioned for its purpose. Part of the network flows must then pass over a larger section of the customer network, and the network load can increase as new services are added, or when services are upgraded. Because of this, it is important to determine whether there is sufficient network and server capacity in the customer network to add new service workflows before they are deployed, or before an existing service is upgraded, ensuring a sufficient level of quality can be guaranteed.

In this article we focus on how the impact of service workflows can be determined, ensuring service workflows do not negatively impact each other's execution. In particular, we determine an impact analysis strategy to evaluate the degree to which a given set of service workflows can be guaranteed in a given network topology. As not all flows are continuously active, the approach is designed to support the sharing of network and server resources using a hierarchically specified resource sharing model. We then evaluate the quality of the resulting solutions using two use cases, and the execution speed of the designed algorithms. For the two evaluation use cases, we find that the developed hierarchical algorithm requires $\pm 42 \%$ and $\pm 52 \%$ less resources than an approach without resource sharing, without any workflow failures occurring during the simulations.
\end{abstract}

Keywords: Resource sharing, Network impact, Service workflows, Cloud offloading

\section{Introduction}

Many service providers install and maintain servers and physical devices on a customer's site to provide a service. These devices and servers work together, ex- 
ecuting workflows that provide the service. When the service must be upgraded the devices must be replaced. Sometimes, the servers have hardware failures or have insufficient processing power, and must be replaced as well. This implies the service provider must dispatch technicians to carry out these changes onsite, making upgrading and maintaining the service offering costly for large customers, and prohibitively expensive for smaller customers. An example of such a service can be found in medical communications, where terminals are installed in hospital rooms and physical management servers are installed in the hospital. These devices are installed and maintained by a third-party service provider.

Traditionally, the on-site devices have been single-purpose, and built specifically to provide a small set of functionality. Currently, there is a trend to migrate to more generic devices, that can support a larger collection of features, and that can be mass-produced. This ensures the service offering can be updated without the need to replace any devices, and makes it possible to activate additional features at a later time. By moving management infrastructure to cloud environments rather than customer-site servers, it becomes easier manage and upgrade the service offering. This also makes it possible to make use of multi-tenancy for the management system, sharing a single management system instance between multiple clients. This can further lower the costs of providing services, as in such a system there no longer has to be a dedicated management instance running at all times for every individual client.

Offering multi-functional hardware and operating them using cloud computing, makes it easier and cheaper to upgrade the offered services, add new functionality, or to enable new services for customers. This in turn makes it possible to offer these services to smaller customers, for whom the cost of upgrading would have been prohibitive. This approach however also has an important downside. Originally, the devices, servers and network were specifically dimensioned to provide a specific service in a controlled environment. In this new configuration three major changes occur: 1) the load on the local network depends on the selected services, which can be changed during the system runtime, 2) as services may make use of cloud infrastructure, some service workflows must pass over a larger part of the customer network, and finally 3 ) as the configuration is done remotely, it is possible to deploy new services at runtime, without the need to visit the customer to install new servers or other devices. New services with varying network impacts can be rolled out more frequently, and these changes impact a larger part of the customer network. Because of this, it is important to create an access filter that can determine the impact on existing deployed services before new services are initialized, or upgraded. This problem is illustrated in Figure 1.

For the considered cases the bottleneck is, both in terms of server capacity and network bandwidth, assumed to be in the customer network and the uplink between the customer network and the cloud environment. This assumption is made because the on-premise network and uplink typically have lower bandwidth than the network within the datacenter, even more so if high-bandwidth cloud instances are used, e.g. Amazon Cluster Compute Instances [1].

We refer to the problem of determining the impact on the network of de- 


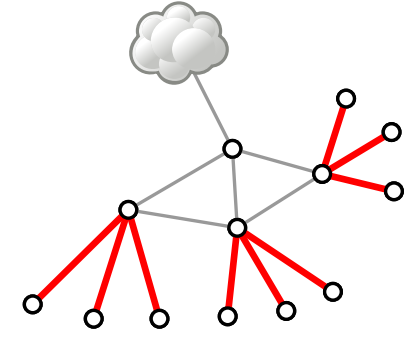

(a) In a network where all services are local, the network communication is restricted to a small part of the network. (The thick red lines represent the service communication flows.)

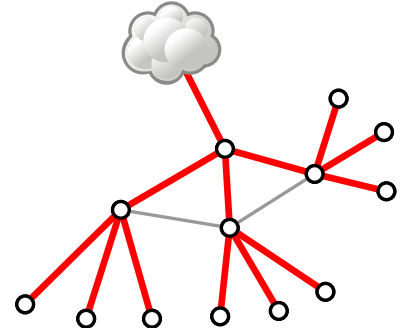

(b) By migrating multiple services to a remote cloud environment, the strain on the client network increases as services are no longer constrained to a small part of the network.

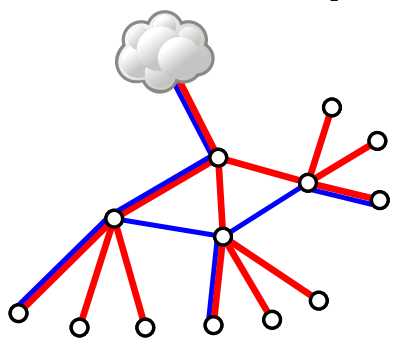

(c) Migrating services to a cloud makes it easier to upgrade them or to add new services as fewer changes are needed on-site. (In this figure, the blue line represents a newly instantiated service.)

Figure 1: An illustration of the impact on a client network of service instantiations, migrations and changes.

ploying services as the Network-Aware Impact Determination (NAID) problem. We previously considered this impact analysis problem for the specific use case where services may either not fail at any cost, or when they are continuously processing information [2]. This implies that services may only be activated if all service workflows can be activated at the same time. When it is rare for all services to be active at the same time, this is an excessive requirement, and greatly limits the number of services that can be permitted on a network. In such a case it would be preferable to support resource sharing within the network, ensuring more services can be activated. This makes it possible to activate more services on the network, but it may also cause resource conflicts. Therefore, an approach is needed where resource sharing is maximized while at the same time the number of resource conflicts is minimized. In this article, we present Shared Resource NAID (SRNAID), an extension to NAID which can be used to hierarchically structure workflows and achieve fine-grained control 
over resource sharing. We also propose a prioritization mechanism based on the NAID model to ensure important flows do not fail in case such resource conflicts occur.

In this article, we focus on network-aware impact analysis with support for resource sharing. We address three research questions: 1) How can resource sharing be incorporated when determining impacts of service workflows on a network during service deployment? 2) How can the resource conflicts occurring due to the sharing of resources be addressed at runtime, ensuring important services are impacted minimally? 3) How does the proposed approach perform, both in terms of service failures and in terms of performance? To this end, we first describe a SRNAID model for determining the impact of adding services with support of network and server resource sharing. To add more fine-grained control over the resource sharing, we define an approach where workflows are grouped in a hierarchy, and where resources are shared at multiple levels of this hierarchy. We then evaluate the SRNAID model using two use cases: a use case based on a Medical Communications (MC) application deployed in a hospital setting and a use case containing a varied collection of generated workflows.

While we focus on a medical communications use case in a partial cloud migration scenario, the designed algorithms can also be applied to general communications use cases, for instance in access control and home automation systems, and in scenarios where data is stored client-side due to compliance rules while (part of) the processing is done in a remote datacenter. Furthermore, the algorithms described may also be of use in multi-cloud deployment scenarios, and as a request admission filter in network function virtualization deployments.

In the next Section, we discuss related work. Afterwards, in Section 3, we discuss the basic NAID problem, which we extend to incorporate support for resource sharing in Section 4. In Section 5 we describe the conflict mitigation used to resolve resource conflicts during the simulation. The simulation setup is described in Section 6, and the results of our evaluations are presented in Section 7. Finally, we state our conclusions in Section 8.

\section{Related work}

Our approach to impact analysis is based on the use of network flow problems [3]. Multi-commodity flow problems [3] are a specific class of network problems that can be used to model various network-problems. Because of this, multi-commodity problems are commonly used in network management for solving various problems such as network routing problems $[4,5,6]$, virtual network allocation [7], and design of fault-tolerant networks [8]. These approaches however work on the network level, and focus on routing flows from one network node to another. We on the other hand add service information to the input network, and focus on service-to-service routing: only the service that is executed matters, not where this service is executed, as long as server resource constraints are respected.

In our previous work [2], we have described a similar network impact analysis framework. In this article, we extend the presented approach and algorithm 
to incorporate support for network resource sharing, significantly reducing the amount of bandwidth needed to accept multiple services and thus increasing the number of services that can be provisioned on a given network. In this article we also propose a hierarchical model to better structure and control the various groups of services between which resources are shared, improving the quality compared to a flat resource sharing approach. The conflict resolution algorithm, which is described in this article and is used in the evaluation of the resource sharing aware algorithm, also makes use of the NAID algorithm described in [2].

Our approach differs from service selection as discussed in [9], where the authors describe an approach for selecting third-party services, such as cloud infrastructure, based on cost and other quality metrics, which focuses on the viewpoint of a client requiring the best-fitting service. We by contrast focus on the viewpoint of a service provider, who needs to select which services can be provided to a client based on the client's network and server capacities.

The approach described in this article has similarities with the application placement problem [10]. Application placement is used to determine the location of applications within networks $[10,11,12]$ or clouds $[13,14,15]$, taking into account the demand for each application. Application placement is used to coordinate applications. This work however focuses on the coordination of service workflows, rather than the management of individual services. In [16] networkaware placement of services is discussed, but the focus is the management of datacenters with specific layouts, so the techniques discussed cannot be directly applied to customer networks. Furthermore, the system assumes bandwidth is the only limitation, ignoring CPU limitations. Our approach however incorporates CPU limitations and can be applied to varying network layouts. In [11] and [17], an application placement algorithm based on a conversion to a network problem is discussed, but the physical network is not taken into account. Our work by contrast specifically focuses on the underlying network.

Our approach further differs from application placement approaches as we assume that the services are already placed. We rather focus on determining which service workflows can successfully execute, given a specific configuration. Thus the approach discussed in this work can be used in conjunction with existing application placement techniques, the application placement techniques being used to determine the service locations, and the SRNAID algorithms to determine the achievable workflows taking into account these service locations. As our techniques can be used to determine bottlenecks, it could also be used to enrich existing placement techniques. Alternatively, it would be possible to extend the described formal model using decision variables to signify instantiation of services on servers to directly use it as an application placement system, but this is not the focus of this article.

The NAID problem is similar to the service matching problem [18]. As in [19], we assume the service specification is known, but while the authors relax the capacity limit to achieve a polynomial time algorithm, we on the other hand focus specifically on these capacity constraints. By focusing on whether the required capacity for offering the services is present in the network, rather 
than on which specific service instances are used within the compositions, we similarly achieve polynomial time algorithms.

The problem we describe is the opposite of network dimensioning $[20,21]$ problems. Network dimensioning is used to determine the required network capacities for a given collection of services. Rather than dimensioning the network to be able to use a given number of services, we focus on determining the services that can be executed given a fixed network configuration. It would be possible to modify the model to use it to determine the minimal required network bandwidth for a given collection of services, and we use this approach to generate difficult problems in our evaluations. This is however not the focus of this article, and does not make it possible to incorporate network survivability, an important aspect for network dimensioning problems.

There are also similarities to the capacity assignment problem [22, 23], where network capacity is assigned to services within networks. We however to not focus on how capacities are divided, but rather on whether there is sufficient capacity at any point in time to provide the service. Additionally, we also consider both the services themselves, and the servers on which they are executing, ensuring there is both sufficient network and service capacity.

\section{Network-Aware Impact Determination}

NAID can be used to determine the impact of workflows on each other. In this context, a workflow is defined as a chain of communicating services between which there is network demand. We have described the NAID problem and proposed various algorithms in our previous work [2]. In this section we present a brief summary of the basic NAID model. Some notational changes are introduced compared to our previous work to enable a more consistent presentation in the remainder of the article where we extend the model to support resource sharing.

We model the NAID problem by transforming the input into a graph problem where both servers and services are represented as nodes within the graph. This makes it possible to model the problem as a variation of the multi-commodity flow problem [4]. An advantage of this approach is that this problem can be solved using a Linear Programming (LP) solver, which as opposed to using Integer Linear Programming (ILP) solvers, do not result in exponential execution time complexity. Additionally, using an LP model, it becomes easier to make modifications to the model, e.g. to support non-linear service configurations. Within the graph, servers are interconnected based on their physical topology. Services are connected to servers on which they are running using two directed edges to represent incoming and outgoing streams of data to the service. We note that in this approach, routers and switches can be represented as server nodes on which no services are running. An example network is shown in Figure 2.

When services communicate, there is a network flow between both, where one service is the source of the flow, while the other is the sink. A network flow from the source service to the sink service is then determined. This flow will 


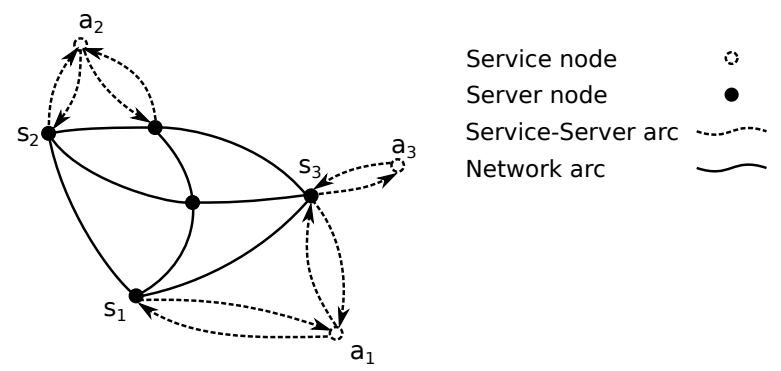

Figure 2: The NAID problem as a flow network. Each service-server arc in the Figure consists of two directed edges, while network arcs can either be bidirectional edges or two directed edges (this is e.g. useful for an uplink where download and upload capacities differ).

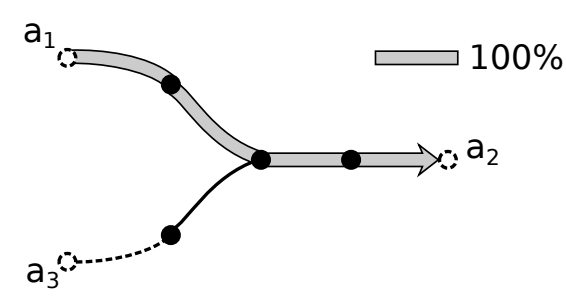

(a) One workflow.

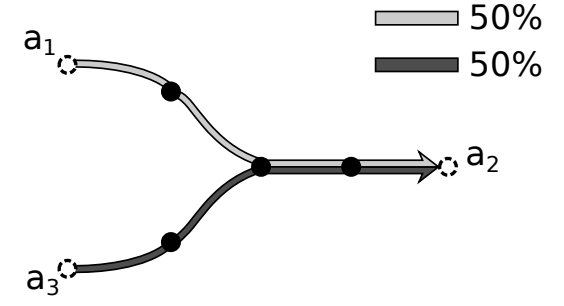

(b) Two workflows.

Figure 3: An illustration of the effect of adding workflows on the guaranteed network share of workflows.

always move over at least one server, even when both the source and sink service are running on the same server. When the services are running on different servers, the flow moves over the physical network between the servers on which the services are running. In the example in Figure 2, a flow between services $a_{1}$ and $a_{2}$ must always move over the physical network (e.g. $a_{1} \rightarrow s_{1} \rightarrow s_{2} \rightarrow a_{2}$ ), while a flow between $a_{1}$ and $a_{3}$ does not have to move over the physical network as both nodes are hosted on server $s_{3}$, but the flow still moves over the server on which the services are running, resulting in the flow $a_{1} \rightarrow s_{3} \rightarrow a_{3}$.

The NAID problem focuses on the available network capacity for services, and the amount of capacity that can be guaranteed for services. Figure 3a shows an illustrative workflow between services $a_{1}$ and $a_{2}$ for which $100 \%$ of the capacity can be guaranteed at all times. Adding a second flow, as illustrated in Figure $3 \mathrm{~b}$ shows the impact of adding a second flow: in this example, only $50 \%$ of the network demand of both services can be guaranteed at all times. This guaranteed network share is formalized as the value $z$, a value that is commonly used for this value in multi-commodity flow literature. This $z$ value represents the share of the demand that can be guaranteed for all workflows within the network. We define a separate $z_{w}$ value for every workflow, making it possible to determine the percentage of the demand for a specific workflow $w$ that can be guaranteed within the given network and service configuration. Whether or 


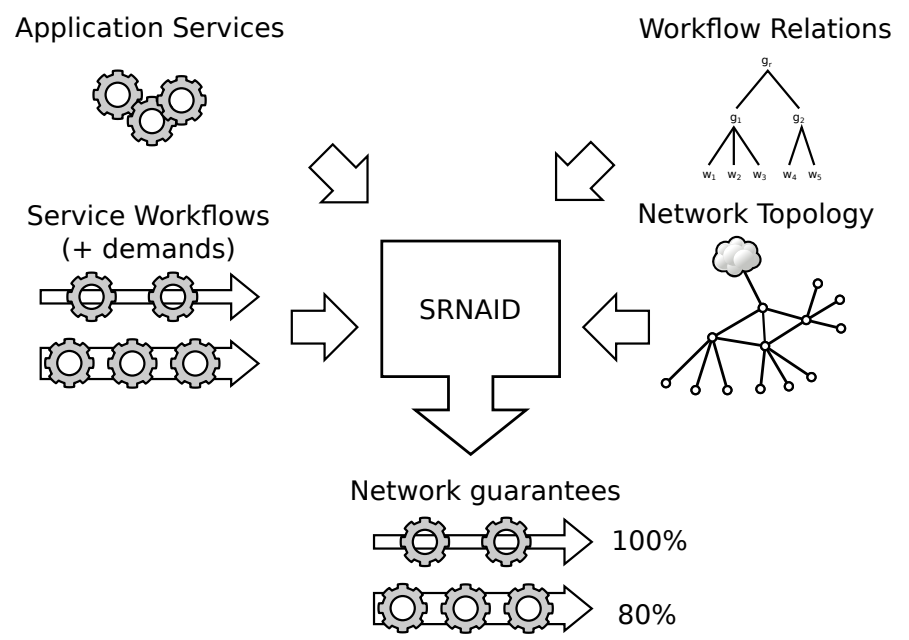

Figure 4: An illustration of the SRNAID problem input and output. The inputs and outputs of the NAID algorithm are similar, but NAID does not take workflow relations into account, making it incapable of sharing resources between workflows resulting in lower achieved network guarantees.

not a $z_{w}$ value of less than $100 \%$ is acceptable for workflows such as those in Figure $3 \mathrm{~b}$ depends on multiple factors:

- If one or both workflows may gracefully degrade, e.g. by reducing video quality if scalable video sources are used, or if delays are acceptable, a lower $z$ value may be permitted.

- If the demand for the workflows fluctuates through time, it may be the case the probability of both requiring their maximum demand at the same time is small. In this scenario, resource conflicts could still occur, but depending on the probability of these conflicts occurring and their consequences the risk may be acceptable.

- If two workflows are guaranteed to never occur at the same time, e.g. as both use the same device at the same time for different purposes, there will never be a problem. While statically it is impossible to guarantee $100 \%$ capacity for both workflows in this scenario without taking this information into account, there will in practice never be resource conflicts making the configuration acceptable.

Making it possible to support these types of workflow interactions is an important motivation for the development of the SRNAID algorithm presented later on in the paper. The inputs and outputs of the SRNAID and NAID algorithms are illustrated in Figure 4. In the remainder of this section we will formalize the NAID algorithm and model. In Section 4 we will then describe the SRNAID algorithm that extends the NAID model, taking workflow relations into account. 


\subsection{Base model: determining service workflow network impact}

The NAID model contains a collection of nodes $\mathcal{N}$ that are either server nodes, contained in a subset $\mathcal{S}$ or application services $\mathcal{A}$. These nodes can be connected using either directed edges, contained in the set $\mathcal{D}$, or bidirectional edges, contained in the set $\mathcal{B}$. These bidirectional edges are implemented as two directed edges, and for every edge $b \in \mathcal{B}$, there is a corresponding edge $\operatorname{cor}(b) \in$ $\mathcal{B}^{\prime}$, the set of corresponding edges. Together, these three collections of edges are contained in a collection of edges $\mathcal{E}$.

A collection of service workflows $\mathcal{W}$ is defined. Every workflow $w \in \mathcal{W}$ is represented as a chain of services and demands, where each service implements a part of the workflow functionality. The workflow starts from an initial service, $a_{1}^{w}$ and goes to the next service until a final service $a_{n}^{w}$ is reached ${ }^{1}$. The bandwidth demand between two services is defined by the variable $d_{i, i+1}^{w}$, which is specified for every pair of services $a_{i}^{w}, a_{i+1}^{w}$ within the workflow as illustrated in Equation (1). Each pair of services within the workflow becomes a separate network flow going from a source to a sink node, which is referred to as a commodity within the multi-commodity flow problem.

$$
w: a_{0} \stackrel{d_{0,1}^{w}}{\longrightarrow} a_{1}^{w} \stackrel{d_{1,2}^{w}}{\longrightarrow} a_{2}^{w} \stackrel{d_{2,3}^{w}}{\longrightarrow} \ldots \stackrel{d_{n-1, n}^{w}}{\longrightarrow} a_{n}^{w}
$$

The approach used to determine the demand between two services of a workflow, $d_{j, j+1}^{w}$, is shown in Equations (2) and (3), and is dependent on a rate between incoming and outgoing flows, $\mathrm{r}_{i n}^{\text {out }}(a)$ which is defined for every service $a$.

$$
\begin{aligned}
& \forall w \in \mathcal{W}: d_{0,1}^{w}=d^{w} \\
& \forall w \in \mathcal{W}: d_{j, j+1}^{w}=d_{j-1, j}^{w} \times \mathrm{r}_{i n}^{\text {out }}\left(a_{j}^{w}\right)
\end{aligned}
$$

The optimization objective is to maximize the share of workflow demand that is allocated. That is, for every workflow $w \in \mathcal{W}$ there is a decision variable $z_{w} \in[0,1]$ which indicates which share of the demand for workflow $w$ that can be provided. If, for a workflow $w, z_{w}=1$, it means that the capacity demanded by it is available to it all of the time. If, on the other hand a lower $z_{w}$ value occurs, such as 0.5 it means that only $50 \%$ of the demand can be guaranteed at all times. This is not necessarily a problem as mentioned in the previous section: NAID does not take resource sharing into account, causing there to potentially be more resources available at runtime. If varying demands during runtime cause more resources to be available, or if the workflow is capable of scaling back resource requirements, the problem may be mitigated at runtime. The objective of the optimization is to maximize these $z_{w}$ values, resulting in the maximum possible quality. This is formulated using the following optimization criterion:

$$
\max \sum_{w \in \mathcal{W}} z_{w}
$$

\footnotetext{
${ }^{1}$ Note that an additional initial service $a_{0}$ is defined. The initial service $a_{0}$ is the same for all workflows, and is used to ensure that each service requiring CPU resources has an input flow, which is used to model CPU load constraints shown further on in this section. For more information concerning the initial service we refer to [2].
} 
The model defines decision variables $\mathrm{f}(e, c)$, representing the network flow over an edge $e \in \mathcal{E}$ for a commodity $c \in \mathcal{C}$. Using these edge flows, the net flow for a specific node and commodity combination can be determined. This net flow is the sum of all incoming flows minus the sum of all outgoing flows, and is shown in Equation (5). For nodes that are not a source or sink, these net flows should be zero, as no flow may be lost within the network. For source nodes there is a negative flow as there is more outgoing flow than incoming flow. Similarly, sink nodes have a positive net flow. Every commodity flow has an incoming flow that is equal to the demand for the commodity, represented as $\mathrm{d}(c)$ which can be calculated as explained previously, multiplied by the share of the total workflow that is achieved, which is represented by $z_{w}$. This is expressed in the flow conservation constraints shown in Equation (6).

$$
\begin{aligned}
& \forall n \in \mathcal{N}: \forall c \in \mathcal{C}: \mathrm{f}(n, c)=\sum_{(m, n) \in E} \mathrm{f}((m, n), c)-\sum_{(n, m) \in E} \mathrm{f}((n, m), c) \\
& \forall n \in \mathcal{N}: \forall c \in \mathcal{C}: \mathrm{f}(n, c)= \begin{cases}-z_{w} \times \mathrm{d}(c) & \text { If } n \text { source of } c \\
z_{w} \times \mathrm{d}(c) & \text { If } n \text { sink of } c \\
0 & \text { Otherwise }\end{cases}
\end{aligned}
$$

Edges between servers are subject to a network link capacity constraint expressed in Equation (7) and Equation (8) for directional and bidirectional edges respectively. These capacities can be measured using existing bandwidth estimation approaches $[24,25,26]$ and are used as an input for the model. Edges between servers and services represent the execution of a service on a server, and are subject to a CPU capacity constraint, which is determined for every server. This constraint is shown in Equation (9); note that only directed edges need to be taken into account here as service-server edges are always directed. In this expression, $\mathrm{r}_{i n}^{C P U}(a)$ represents a ratio converting the used network bandwidth to a measure of CPU use.

$$
\begin{aligned}
& \forall e \in \mathcal{D} \cap \mathcal{S} \times \mathcal{S}: \sum_{c \in C} \mathrm{f}(e, c) \leq \operatorname{cap}(e) \\
& \forall e \in \mathcal{B} \cap \mathcal{S} \times \mathcal{S}: \sum_{c \in C} \mathrm{f}(e, c)+\sum_{c \in C} \mathrm{f}(\operatorname{cor}(e), c) \leq \operatorname{cap}(e) \\
& \forall s \in \mathcal{S}: \sum_{(s, a) \in \mathcal{D}}\left(\mathrm{r}_{i n}^{C P U}(a) \times \sum_{c \in C} \mathrm{f}((s, a), c)\right) \leq C P U_{s}
\end{aligned}
$$

Workflows are a sequence of different execution steps: the incoming flow in a service is processed, and results in an outgoing flow from the service that is sent the next service. This is expressed in the workflow chain relation constraint shown in Equation (10).

$$
\forall w \in \mathcal{W}: \mathrm{r}_{i n}^{\text {out }}\left(a_{n}^{w}\right) \times \mathrm{f}\left(\left(s, a_{n}^{w}\right), c_{i}^{w}\right)=\mathrm{f}\left(\left(a_{n}^{w}, s\right), c_{i+1}^{w}\right)
$$

The service-server edges may only be used to provide flow going to the specific services, and may not be used for any other services. Because of this, two 
additional constraints, Equation (11) and Equation (12) are added to restrict respectively outgoing and incoming flows. In this formulation, $a_{1}$ and $a_{2}$ represent the source and the sink service of the commodity $c$.

$$
\begin{aligned}
& \forall c \in \mathcal{C}: \forall a \in \mathcal{A}: \forall s \in \mathcal{S}: \mathrm{f}((a, s), c)=0 \text { (unless } a=a_{1} \text { ) } \\
& \forall c \in \mathcal{C}: \forall a \in \mathcal{A}: \forall s \in \mathcal{S}: \mathrm{f}((s, a), c)=0 \text { (unless } a=a_{2} \text { ) }
\end{aligned}
$$

An additional artificial service, $a_{0}$ is also added to ensure all workflows have an input flow. This is needed to correctly specify the CPU capacity constraint. As this service is artificial, the flow for any workflow commodity $\left(a_{0}, a_{w_{1}}\right)$ which starts in this service may not pass over server-server links. To enforce this, the constraint in Equation (13) is added for all $e \in E$ of the type $\left(s_{1}, s_{2}\right) \in S^{2}$, and all commodities $c \in C$ for which the flow starts in $a_{0}$.

$$
\forall c \in \mathcal{C}: \forall\left(s_{1}, s_{2}\right) \in \mathcal{S} \times \mathcal{S}: f\left(\left(s_{1}, s_{2}\right), c\right)=0
$$

For performance reasons, we only consider the CPU constraints for servers active within the customer network, and not for physical devices or cloud nodes. This is possible as the considered physical devices are built to provide a limited collection of specific services, thus they are designed to be capable of providing these services. Within the cloud, additional computational capacity can be requested on-demand when insufficient resources are present.

\subsection{Non-workflow service graphs}

Within this article we focus on service workflows. In practice, coordinating services can however not always be represented as linear workflows, and arbitrary service configurations may need to be supported. These configurations can be represented as a graph where the nodes are services and the edges are the capacity demands between the services. For this reason, we refer to them as service graphs. A workflow is a service graph where a source and sink node have one outgoing and one incoming edge respectively, while all other nodes have both an incoming and an outgoing edge. Thus, service graphs are a generalization of the service workflow concept.

The approach discussed previously can be extended to support such configurations: in the model, it is possible to create multiple flows from a single source node, which is all that is needed to add support for arbitrary service graphs. Table 1 shows how three service graphs can be represented within the flow based model: the workflow (as presented in the previous section), a graph where one service interacts with multiple services, and a star service graph. The same approach can be used to represent more complex non-linear service graphs.

In general, these service graphs can be defined by defining a $\mathrm{r}_{i n}^{\text {out }}\left(s_{a}, s_{b}\right)$ variable for all services $s_{a}$ and $s_{b}$ that are connected in the service graph. This variable specifies the network demand between the two services. As illustrated in Table 1, this value can then be specified based on the incoming flow within the service $s_{a}$. If multiple flows enter a service, the outgoing flow can either be specified based on one of the incoming flows, or the sum of the incoming flows. 
Table 1: An illustration of how various service graphs can be represented within the flow-based model. The defining variables column shows which input variables are needed to characterize the flow.

\begin{tabular}{clc}
\hline Service graph & Flow specification & Defining Variables \\
\hline & $d_{0,1}=d$ & $d$ \\
$\mathrm{~s}_{1} \rightarrow \mathrm{s}_{2} \rightarrow \mathrm{s}_{3} \rightarrow \mathrm{s}_{4}$ & $d_{1,2}=d_{0,1} \times \mathrm{r}_{i n}^{\text {out }}\left(s_{1}\right)$ & $\mathrm{r}_{i n}^{\text {out }}\left(s_{1}\right)$ \\
& $d_{2,3}=d_{1,2} \times \mathrm{r}_{i n}^{\text {out }}\left(s_{2}\right)$ & $\mathrm{r}_{i \text { iut }}^{\text {out }}\left(s_{2}\right)$ \\
& $d_{3,4}=d_{2,3} \times \mathrm{r}_{\text {in }}^{\text {out }}\left(s_{3}\right)$ & $\mathrm{r}_{i n}^{\text {out }}\left(s_{3}\right)$ \\
\hline $\mathrm{s}_{1} \rightarrow \mathrm{s}_{2} \rightarrow \mathrm{s}_{3}$ & $d_{0,1}=d$ & $d$ \\
$\downarrow$ & $d_{1,2}=d_{0,1} \times \mathrm{r}_{i n}^{\text {out }}\left(s_{1}\right)$ & $\mathrm{r}_{\text {in }}^{\text {out }}\left(s_{1}\right)$ \\
$\mathrm{s}_{4}$ & $d_{2,3}=d_{1,2} \times \mathrm{r}_{i n}^{\text {out }}\left(s_{2}, s_{3}\right)$ & $\mathrm{r}_{i n}^{\text {out }}\left(s_{2}, s_{3}\right)$ \\
& $d_{2,4}=d_{1,2} \times \mathrm{r}_{\text {in }}^{\text {out }}\left(s_{2}, s_{4}\right)$ & $\mathrm{r}_{\text {in }}^{\text {out }}\left(s_{2}, s_{4}\right)$ \\
\hline $\mathrm{s}_{1} \leftarrow \mathrm{s}_{2} \rightarrow \mathrm{s}_{3}$ & $d_{0,2}=d$ & $d$ \\
$\downarrow$ & $d_{2,1}=d_{0,1} \times \mathrm{r}_{i n}^{\text {out }}\left(s_{2}, s_{1}\right)$ & $\mathrm{r}_{i n}^{\text {out }}\left(s_{2}, s_{1}\right)$ \\
$\mathrm{s}_{4}$ & $d_{2,3}=d_{0,2} \times \mathrm{r}_{i n t}^{\text {out }}\left(s_{2}, s_{3}\right)$ & $\mathrm{r}_{i n t}^{\text {out }}\left(s_{2}, s_{1}\right)$ \\
& $d_{2,4}=d_{0,2} \times \mathrm{r}_{\text {in }}^{\text {out }}\left(s_{2}, s_{4}\right)$ & $\mathrm{r}_{\text {in }}^{\text {out }}\left(s_{2}, s_{4}\right)$ \\
\hline
\end{tabular}

Arbitrary service graphs can therefore be supported by the model. Within the evaluations in this article we however focus specifically on linear service workflows.

\section{Shared Resource NAID}

The model discussed in the previous section is useful in a context where service workflows are continuously transmitting or in contexts where a very high reliability is needed. For many use cases, not all services will be active at the same time, making it preferable to support resource sharing. This ensures more services will be allowed to be deployed, at the risk of resource conflicts occurring. In this section we discuss how resource sharing can be added to the NAID model by allowing workflows to partially ignore each others resource requirements. First, we will discuss network resource sharing, and subsequently we will discuss server resource sharing. In both cases we will make use of a hierarchical specification of services, where similar service workflows are grouped together. This hierarchical approach makes it possible to group workflows with similar properties together making it much easier to specify the relations between workflows, and making it possible to have fine-grained control over the degree by which workflows may ignore each others resource requirements.

\subsection{Adding resource sharing: network edge sharing}

To achieve network edge capacity sharing, we make use of two major changes to the previously specified NAID model: 1) capacity constraints are expressed 
per workflow and per edge, rather than just per edge, and 2) to achieve a finergrained control over the sharing of network capacity, the workflows are grouped in a hierarchy.

Within the NAID model, edge capacities are expressed using Equation (7). This expression is equivalent to Equation (14), where it is expressed for every workflow. By then adding an overprovisioning factor $O F$, as illustrated in Equation (15), a rudimentary system for sharing resources on edges can be defined. When $O F=1$, no network resource sharing will occur, ensuring that the network is overprovisioned and that all resources will be able to be executed at the same time. If $O F<1$ sharing of network capacity between service workflows occurs.

$$
\begin{gathered}
\forall e \in \mathcal{E}: \sum_{c \in C} \mathrm{f}(e, c) \leq \operatorname{cap}(e) \\
\forall e \in \mathcal{E}, w \in \mathcal{W}: \sum_{c \in w} \mathrm{f}(e, c) \leq \operatorname{cap}(e)-\sum_{c^{\prime} \notin w} \mathrm{f}\left(e, c^{\prime}\right) \\
\forall e \in \mathcal{E}, w \in \mathcal{W}: \sum_{c \in w} \mathrm{f}(e, c) \leq \operatorname{cap}(e)-O F \times \sum_{c^{\prime} \notin w} \mathrm{f}\left(e, c^{\prime}\right)
\end{gathered}
$$

Using this approach, $O F$ determines the degree to which other services are taken into account during optimization. An $O F$ of 0 ensures that all other flows are ignored, while an $O F$ of 1 results in the original equation of the NAID model where all workflows are fully taken into account and no sharing of resources occurs.

This approach is however not sufficient as all workflows are treated equally, offering only limited control. In practice different workflows can have different characteristics and probabilities of interfering with each other. To resolve this, we define a hierarchy containing workflows and groups of workflows. The leaves of this hierarchy are the workflows themselves, while inner nodes of the hierarchy group various workflows or other nodes together. An example is shown in Figure 5a, in this example a group $g_{1}$ contains three workflows $w_{1}, w_{2}$, and $w_{3}$,

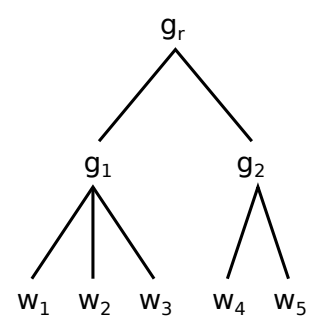

(a) Workflows grouped hierarchically.

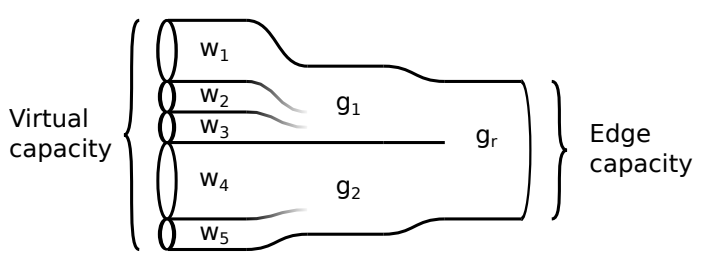

(b) Network edge capacity sharing on different hire erarchically specified levels.

Figure 5: Hierarchical capacity sharing between edges ensures more capacity is available for individual workflows than physically present. The various workflows within a group share resources based on the characteristics of the group, such as the probability of occurring at the same time. 
group $g_{2}$ contains two workflows, and the root group $g_{r}$ contains two groups $g_{1}$ and $g_{2}$. Every element of the hierarchy is assigned an individual capacity for every edge, and resource sharing occurs within individual groups. This concept is illustrated in Figure 5b, and makes it possible to control the process of resource sharing for every service and on multiple levels.

Formally, we define a collection of hierarchy nodes $\mathcal{H}$ that contains both all workflows, contained in the collection $\mathcal{W}$, and all groups, contained in a collection $\mathcal{G}$. Each group $G \in \mathcal{G}$ can contain multiple workflows or flow groups. Every $h \in \mathcal{H}$, except for the root, is contained in exactly one group, creating the hierarchy. For the example in Figure 5a this results in the following collections:

$$
\begin{aligned}
\mathcal{W} & =\left\{w_{1}, w_{2}, w_{3}, w_{4}, w_{5}\right\} \\
\mathcal{G} & =\left\{\left\{w_{1}, w_{2}, w_{3}\right\},\left\{w_{4}, w_{5}\right\},\left\{\left\{w_{1}, w_{2}, w_{3}\right\},\left\{w_{4}, w_{5}\right\}\right\}\right\}
\end{aligned}
$$

To make use of capacity constraints per group, we first define the capacity assigned to a workflow on an edge. This is shown in Equation (16) for directed edges and in Equation (17) for bidirectional edges.

$$
\begin{aligned}
& \forall e \in \mathcal{D}, w \in \mathcal{W}: \operatorname{cap}(e, w)=\sum_{c \in w} \mathrm{f}(e, c) \\
& \forall e \in \mathcal{B}, w \in \mathcal{W}: \operatorname{cap}(e, w)=\sum_{c \in w}(\mathrm{f}(e, c)+\mathrm{f}(\operatorname{cor}(e), c))
\end{aligned}
$$

We also define a new decision variable $\operatorname{cap}(e, g)$ for all groups $g \in \mathcal{G}$ and all edges $e \in \mathcal{E} \cup \mathcal{B}$. Finally, a constraint is defined for every group in the hierarchy, linking the capacity assigned to its children to its own capacity. For this we use an expression based on that of Equation (15), but rather than defining it for the total set of commodities $\mathcal{C}$ it is used within individual groups. The resulting expression is shown in Equation (18). This expression makes use of a factor $O F_{G}$ which is defined within a group. Every group can be assigned a different $O F_{G}$ value, depending on characteristics of the group.

$$
\forall G \in \mathcal{G}, h \in G, e \in \mathcal{D} \cup \mathcal{B}: \operatorname{cap}(e, h) \leq \operatorname{cap}(e, G)-O F_{G} \times \sum_{h^{\prime} \in G \backslash\{h\}} \operatorname{cap}\left(e, h^{\prime}\right)
$$

As mentioned previously, the workflows and groups form a hierarchy. The root group, $G_{r}$, has a capacity which is limited by the edge capacity. This is expressed in Equation (19). Additionally, a constraint to ensure the final capacity remains larger than that used for individual workflows is also added. This constraint, shown in Equation (20) ensures that no individual flow can ever be larger than the network edge capacity.

$$
\begin{array}{r}
\mathrm{f}\left(e, G_{r}\right)=\operatorname{cap}(e) \\
\forall c \in \mathcal{C}: \mathrm{f}(e, c) \leq \operatorname{cap}(e)
\end{array}
$$




\subsection{Adding resource sharing: server capacity sharing}

The server resource formulation which is included for all $s \in \mathcal{S}$ can be reformulated in a similar way to the transformation of network capacity limits. For this we first define an expression $L^{C P U}(s, w)$ which, for a workflow $w \in \mathcal{W}$, determines the CPU load on the server:

$$
L^{C P U}(s, w)=\sum_{(s, a) \in E}\left(\mathrm{r}_{i n}^{C P U}(a) \times \sum_{c \in w} \mathrm{f}((s, a), c)\right)
$$

By modifying the NAID model CPU constraint, Equation (9), to incorporate this $L^{C P U}$ formulation, Equation (21) can be obtained. This formulation is similar to that shown previously in Equation (21), and like in the previous section, an overprovisioning $O F$ factor can be added as expressed in Equation (22).

$$
\begin{array}{r}
\forall s \in \mathcal{S}: \sum_{(s, a) \in E}\left(\mathrm{r}_{i n}^{C P U}(a) \times \sum_{c \in C} \mathrm{f}((s, a), c)\right) \leq C P U_{s} \\
\forall s \in \mathcal{S}: \sum_{(s, a) \in E}\left(\mathrm{r}_{i n}^{C P U}(a) \times \sum_{w \in \mathcal{W}} \sum_{c \in w} \mathrm{f}((s, a), c)\right) \leq C P U_{s} \\
\forall s \in \mathcal{S}: \sum_{w \in \mathcal{W}} \sum_{(s, a) \in E}\left(\mathrm{r}_{i n}^{C P U}(a) \times \sum_{c \in w} \mathrm{f}((s, a), c)\right) \leq C P U_{s} \\
\forall s \in \mathcal{S}: \sum_{w \in \mathcal{W}} L^{C P U}(s, w) \leq C P U_{s} \\
\forall s \in \mathcal{S}: \forall w \in \mathcal{W}: L^{C P U}(s, w) \leq C P U_{s}-\sum_{w^{\prime} \in \mathcal{W} \backslash\{w\}} L^{C P U}\left(s, w^{\prime}\right) \\
\forall s \in \mathcal{S}: \forall w \in \mathcal{W}: L^{C P U}(s, w) \leq C P U_{s}-O F \times \sum_{w^{\prime} \in \mathcal{W} \backslash\{w\}} L^{C P U}\left(s, w^{\prime}\right)
\end{array}
$$

Analogous to how this was done in the previous section, we can now define this constraint for hierarchically specified groups. For every group $G \in \mathcal{G}$ and

every server $s \in \mathcal{S}$ we define a new decision variable $L^{C P U}(s, G)$ representing the CPU capacity assigned to a group. The capacity of the root group $G_{r}$ is defined as equal to the CPU capacity of the server:

$$
L^{C P U}\left(s, G_{r}\right)=C P U_{s}
$$

We now add flow group capacities and an overprovisioning factor, $O F_{G}$, for every group, similar to how this was done in this in Section 4.1. The resulting constraint is shown in Equation (24).

$\forall G \in \mathcal{G}, h \in G, s \in \mathcal{S}: L^{C P U}(s, h) \leq L^{C P U}(s, G)-O F_{G} \times \sum_{h^{\prime} \in G \backslash\{h\}} L^{C P U}\left(s, h^{\prime}\right)$ 


\section{Runtime conflict management}

When resources are shared between workflows, and the above SRNAID algorithm is used to determine whether services are allowed to be deployed, it is possible for resource conflicts to occur. These resource conflicts occur when, at a given time during the execution, there is more demand for resources than there are available. How these resource conflicts are handled depends on the services and workflows themselves, and these conflicts can be handled in multiple ways: 1) the workflows may execute in a reduced quality mode (e.g. by using lower bitrate video and audio), 2) the workflow execution may be delayed until there is sufficient capacity for its execution, or 3) the workflow may fail causing it to not be executed at all. Only the last two approaches can be used generically, and often delaying workflows may cause unacceptable delays causing them to fail as well. Therefore we focus on the last scenario. Often, some workflows are more important than others, making it important to develop a strategy for dynamically failing less important workflows to prevent these more important flows from failing. Whenever a new workflow is started, the system state must be reevaluated: it must be determined whether the workflow can be added to the system, or whether it conflicts with already existing workflows. If there is a conflict this must be resolved. To achieve this, we make used of a modified NAID algorithm which is used in a workflow addition algorithm.

\subsection{Adding quality levels: class-aware NAID}

An important part of the conflict mitigation algorithm is a class-aware NAID algorithm. This algorithm is based on the NAID model without resource sharing outlined in Section 3, but incorporates the concept of a workflow class, indicating the importance of the workflow. We refer to this algorithm as $N A I D^{*}$. By associating a class with workflows, we can prioritize the execution of important workflows compared to other, less important, workflows.

With every workflow $w \in \mathcal{W}$ we associate a value class $(w)$ which represents the quality level or importance of the flow. A workflow with a low class value is deemed more important than a workflow with a higher class value, with the highest workflow quality level being 0 . The $N A I D^{*}$ algorithm takes one additional parameter compared to the regular NAID algorithm: $w_{\text {new }}$, the workflow which is the newest workflow to be added to the system. Additionally, a collection of new constraints, shown in Equation (25), is added:

$$
\forall w \in \mathcal{W}: \operatorname{class}(w) \leq \operatorname{class}\left(w_{\text {new }}\right) \Rightarrow z_{w}=1
$$

This constraint ensures that, all workflows of the same or higher priority will be fully provided, and only lower-quality workflows may still fail. When running the $N A I D^{*}$ algorithm, there are three possible outcomes:

1. Every $z_{w}$ value is 1 . This implies all of the requested workflows can be provided.

2. Some $z_{w}$ values are less than 1 . In this case some lower-priority workflows can not be provisioned correctly, but all of the workflows of the quality of $w_{n e w}$ and all workflows of higher quality can be fully provisioned. 
3. The resulting model is infeasible and no configuration can be determined. This implies that it is impossible to provide the workflows of the current quality level and higher quality, and that at least one of these flows must be removed. In a runtime scenario where the $N A I D^{*}$ algorithm is executed iteratively this is an interesting case, as if at a given point in time the result of $N A I D^{*}$ execution is infeasible, and at the previous point it was feasible the infeasibility can be traced back to the last added workflow $w_{\text {new }}$.

Note that this algorithm does not take resource sharing into account as only workflows that are active at a given point in time are taken into account.

\subsection{Workflow addition algorithm}

To add workflows to a given configuration, Algorithm 1 is used. The algorithm takes as input a set of active workflows, and a new workflow that is to be placed, and returns three possible values: 1) $O K$, which indicates that the workflow can be added without impacting the active workflows; 2) Fail, which indicates that the workflow can not be added, as it would negatively impact high-priority active workflows; and 3) Abort(abortedFlows), which indicates

that the workflow can indeed be added, but that to do so the returned set of lower priority workflows must be aborted, causing those workflows to fail.

Data: active: Set of currently active workflows

Data: new: New workflow to be placed

Result: Return state

1 Solve $N A I D^{*}$ for workflows active $\cup\{$ new $\}$;

2 if Infeasible then

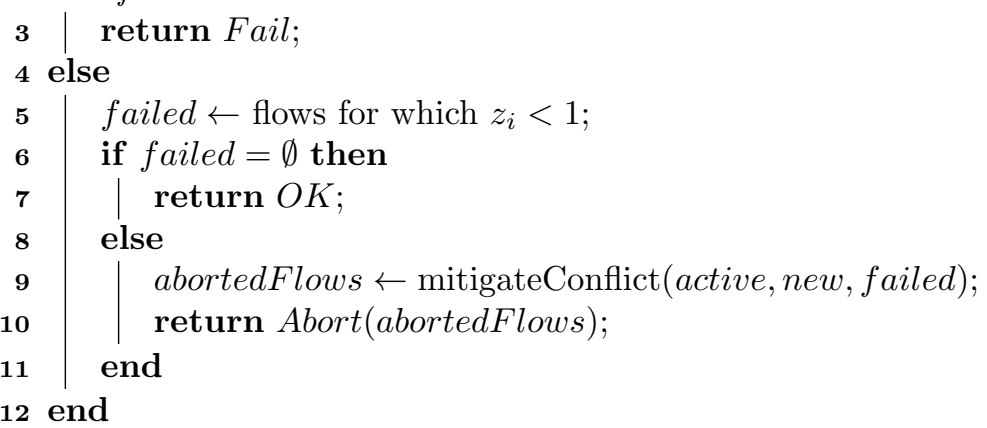

Algorithm 1: The doPlace function. The algorithm can return three states: $O K$ indicating the new workflow it can run; Fail indicating the new workflow can not run; and Abort(abortedFlows) indicating the new flow can run, but that some active flows, those contained in the set abortedFlows, must be aborted to do so.

The doPlace function first solves the $N A I D^{*}$ algorithm which was mentioned in the previous section. If the $N A I D^{*}$ formulation is infeasible, this implies that it is impossible to achieve a configuration where all workflows with the 
quality level of new and higher are successful, even if all lower quality flows are eliminated. Thus, the new flow may not be added and Fail is returned.

If $N A I D^{*}$ succeeds, the workflows for which $z_{w}<1$ are determined. If there are none, $O K$ is returned and the algorithm finished. If some workflows fail, the conflict will have to be mitigated. These failed flows are stored in the set fail. At this stage it would be possible to return all the failed workflows and abort their execution, which would result in a feasible configuration. This configuration could however be suboptimal in two ways: 1) it could occur that multiple workflows are not entirely successful in the $N A I D^{*}$ solution, but that removing only a single flow is sufficient to achieve a feasible configuration; or 2 ) it may be possible to resolve the conflict by aborting lower-priority flows than those present in the set fail. For these reasons a separate mitigateConflict function is defined.

Data: active: Set of currently active workflows

Data: new: New workflow to be placed

Data: failed: The flows that failed in a previous placement

Result: fail: The workflows that must be removed to yield a feasible configuration

1 worst $\leftarrow$ workflow with lowest $z_{i}$ in failed;

2 reversedState $\leftarrow$ doPlace $($ active $\cup\{$ new $\} \backslash\{$ worst $\}$, worst $)$;

3 if reversedState $=$ Abort (abortedFlows) then

/* A feasible configuration exists by removing the selected set of workflows

$4 \quad$ return abortedFlows;

5 else if reversedState $=$ Fail then

/* The workflow worst is incompatible with higher-class workflows, it must be completely removed */

$6 \quad$ restrictedPlace $\leftarrow$ doPlace $($ active $\backslash\{$ worst $\}$, new $)$;

$7 \quad$ if restrictedPlace $=$ Abort (abortedFlows) then

$\mid$ /* Aborting the resulting flows and worst results in a feasible configuration

\begin{tabular}{l|r|}
8 & return abortedFlows $\cup\{$ worst $\} ;$ \\
9 & else if restrictedPlace $=O K$ then
\end{tabular}

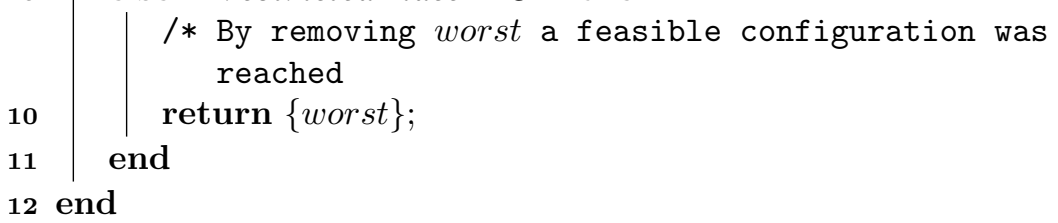

/* By removing worst a feasible configuration was reached

12 end

Algorithm 2: The mitigateConflict function used to determine the set of workflows that are to be removed to resolve a resource conflict.

Algorithm 2 shows how resource conflicts are mitigated. First, the algorithm tries to resolve the conflict by removing the workflow that achieves the lowest $z_{w}$ value, referred to as worst, and trying to place that workflow with doPlace, 
assuming all other workflows, including the new one, are active. This can result in two possible responses ${ }^{2}$ :

1. Abort: It is possible that the recursive call leads to a list of failed flows that must be aborted. By aborting these flows a feasible configuration can be determined.

2. Fail: This implies that workflow worst can not be placed in the configuration. We then retry adding the original workflow new using doPlace, but remove workflow worst from the active set of workflows.

\subsubsection{Addition algorithm termination}

The doPlace function makes use of the mitigateConflict function and vice versa, creating a recursive structure. To ensure the execution always terminates, we consider two values associated with every invocation of doPlace and mitigateConflict: class (new), the class of the newly added workflow, and $\mid$ active $\mid$, the number of elements contained within the set active.

We first note that, if mitigateConflict is invoked from doPlace, the same active and new as used in the doPlace invocation are used, so we can focus specifically on the mitigateConflict function. The doPlace function is invoked at two points in mitigateConflict:

- The doPlace invocation on Line 2 tries to place the workflow worst. As worst had a $z_{i}$ value less than 1 , this implies worst has a lower priority than new, and thus a higher class, as otherwise $N A I D^{*}$ would have assigned $z_{i}$ value 1 to it because of the constraint in Equation (25). This new invocation of doPlace thus works on a class of class(worst) $>$ class $(n e w)$. The number of workflows that is to be placed, $\mid$ active $\mid$ remains the same in this invocation.

- The doPlace invocation on Line 6 is executed using a set active $\backslash\{$ worst $\}$. As the resulting set contains one less item, it is of lower cardinality than in the previous iteration. As the workflow new that is placed is the same, class (new) remains the same for the invocation.

As the number of workflows that can exists is finite, there is a workflow $w_{m c}$ with the maximal class value $\operatorname{class}\left(w_{m c}\right)$ and thus the lowest priority. The minimal cardinality of a set is that of the empty set: $|\emptyset|=0$. Every invocation of mitigateConflict either increases class (new) by at least one in the next invocation of doPlace, or decreases $\mid$ active $\mid$ by one. As there is an upper limit to class $(n e w)$ and a lower limit to $\mid$ active $\mid$ the algorithm is guaranteed to terminate in at most $O(a+c)$ steps, where $a=\mid$ active $\mid$, the number of active workflows, and $c=\operatorname{class}\left(w f_{m c}\right)-\operatorname{class}(n e w)$, the number of workflow classes that exist that are higher than that of new.

\footnotetext{
${ }^{2}$ The exit state $O K$ of the doPlace function can not occur here as otherwise conflict mitigation would not have been initiated in the first place.
} 


\section{Evaluation approach}

The algorithms presented in this article were implemented using Scala [27], the models were implemented using the CPLEX [28] LP solver. To evaluate the approach we make use of two evaluation scenarios, where we evaluate the quality of the resulting network using discrete event simulation: a Medical Communications (MC) use case and a randomized scenario.

\subsection{Medical communications use case}

MC applications provide multiple communication services within hospitals and other care facilities such as nursing homes. While the cloud has many benefits related to manageability and scalability, a full migration is impossible as the services depend on locally installed hardware terminals. An important side effect of a cloud migration is the increasing impact on the client network, which often uses older hardware and has only limited capacity. Despite this, it is vital that services can be executed uninterrupted, making it important to determine whether services will impact each other before they are deployed. For this, we use the SRNAID approach. The considered MC use case provides three separate services, Nurse Call (NC), Voice over IP (VoIP) and video. The primary and most important functionality is $\mathrm{NC}$, which is provided using terminals and buttons installed in rooms that can be used by patients to call hospital personnel. This important service is subject to strict quality and compliance rules, but requires only limited amounts of network bandwidth. VoIP and video are also offered as add-on services, but while they require significantly more network bandwidth, the impact of failing to provide the service is less severe. Within this system, various workflows exist to provide the three services. Each of these workflows represents a single part of the service, e.g. a patient initiating a $\mathrm{NC}$ or a nurse initiating a VoIP call.

To provide the services, three types of devices are installed at the customer's site: controllers, handheld mobile devices and terminals. These devices communicate with each other, and with a remote cloud environment. The customer

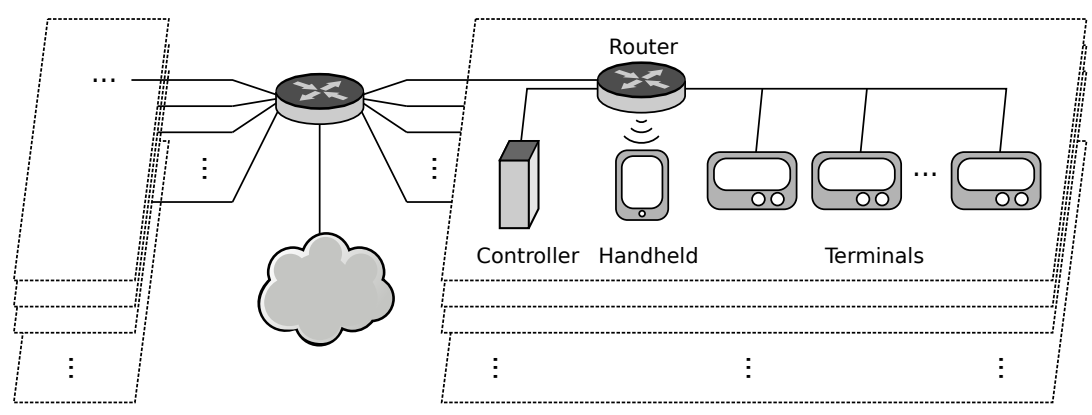

Figure 6: The network set-up used in the evaluation environment. The environment models two buildings with multiple floors and a collection of end-user devices on every floor. The entire set-up is connected to a remote cloud environment. 
Table 2: MC evaluation scenario workflows.

\begin{tabular}{|c|c|c|c|}
\hline Name & Load & Start & Duration \\
\hline $\mathrm{NC}$ & $2 \mathrm{KBps}$ & $\begin{array}{l}\text { Exponential } \\
\quad \lambda=1 / h\end{array}$ & $\begin{array}{c}\text { Normal } \\
\mu=1 \mathrm{~s}, \sigma=100 \mathrm{~ms}\end{array}$ \\
\hline VoIP & $10 \mathrm{KBps}$ & $\begin{array}{l}\text { Exponential } \\
\quad \lambda=1 / h\end{array}$ & $\begin{array}{l}\text { Erlang } \\
\mu=30 s, \sigma^{2}=50 s^{2}\end{array}$ \\
\hline Video & $100 \mathrm{KBps}$ & $\begin{array}{l}\text { Exponential } \\
\lambda=1 / 5 h\end{array}$ & $\begin{array}{c}\text { Erlang } \\
\mu=50 s, \sigma^{2}=90 s^{2}\end{array}$ \\
\hline
\end{tabular}

network layout consists of two buildings, with different floors. Routers are provided per-floor and they are connected to a central uplink. The result is a tree-based structure, where inner nodes represent the network infrastructure and routers, while the leaf nodes are controllers, handhelds and terminal nodes. The network set-up is shown in Figure 6. For the quality evaluations a small hospital is used with two buildings, three floors per building, and ten leaf nodes.

Within the system, there are three types of service workflows, which are shown in Table 2. The workflows connect end user terminals with a local controller, and for compliance and logging reasons a reduced version of the stream is sent to a cloud-hosted management component. For each of the workflows we determine a network load, a start distribution and a duration distribution. The start distribution determines the next time when the workflow will be started, while the duration distribution determines how long a workflow will require resources once it has been activated.

The start distribution of each of the workflows is exponential which is chosen as it describes events that occur continuously and independently at a defined rate. For NC, the duration of the workflow is normally distributed as this is an automated process For the other workflows an Erlang distribution is used, as the distribution of the duration of VoIP and video workflows are similar to that of phone calls, for which an Erlang distribution is a good fit. The parameters for the various distributions are based on input from industry partners. These parameters can vary based on the type of facility, as e.g. hospitals typically have more frequent invocations compared to retirement homes.

The service hierarchy used in the SRNAID algorithm contains three levels. There is the root level root, which contains three groups for the three service types, $N C, V o I P$ and Video. Finally, the three groups each contain all of the workflows that are defined for the respective services.

\subsection{Generated use case}

We also consider a second use case containing a larger variety of different randomly generated workflows to determine how the algorithms behave when there is a higher variety in workflows and when data and server intensive workflows interact. In this scenario, we generate a large collection of workflows which we deploy on the previously discussed client network. These workflows have random length and load characteristics, and can be divided into two types: Server 
Table 3: Parameters used in the generated evaluation scenario workflows. Values within ranges are chosen at random using a uniform distribution.

\begin{tabular}{lll}
\hline & Parameters \\
\hline Number of workflows & 50 of each type \\
Flow length & Chosen using uniform distribution from $[2,8]$ \\
Occurence frequency & $\begin{array}{l}\text { Exponential distribution. On average } \alpha \text { times per } \\
\text { interval } t \text { with } t \in[30,90] \text { minutes. }\end{array}$ \\
Duration & $\begin{array}{l}\text { Normal distribution. } \mu \in[10,60] \text { seconds, } \sigma=10 \\
\text { seconds. }\end{array}$ \\
\cline { 2 - 3 } & Server Intensive & Data Intensive \\
\cline { 2 - 3 } Network demand & {$[1,50] \mathrm{MBps}$} & {$[10,500] \mathrm{MBps}$} \\
Server demand & {$[100,1000] \mathrm{MHz}$} & {$[10,100] \mathrm{MHz}$} \\
\hline
\end{tabular}

Intensive (SI) workflows that require many server resources and limited network resources, and Data Intensive (DI) workflows that require more network resources and less server resources.

The parameters used for both workflow types are shown in Table 3. Every workflow is generated with randomized length and resource requirements. For every service contained in a workflow, the number of nodes on which it may run is chosen at random using a non-negative normal distribution with mean 1 and $\sigma=10$ ensuring it can only run on a limited number of servers. There is a $30 \%$ probability that a service may be allocated on the cloud, the other nodes on which the service may run are selected at random from the edge nodes present in the network. The occurrence frequency of the workflows is exponential and depends on a parameter $\alpha$ representing the average number of occurrences within an interval of duration $t$. The $\alpha$ parameters are equal for all workflows of the same type, and are thus specified separately for SI and DI workflows, represented as $\alpha_{S I}$ and $\alpha_{D I}$. The time interval $t$ is chosen randomly for every workflow. By modifying the $\alpha$ parameters, the occurrence frequency of DI and SI workflows can be modified. Half of the workflows of each type are marked as a high priority workflow, the other flows are marked as low priority.

Like in the MC scenario, the service hierarchy in the generated scenario contains three levels. At the top of the tree, there is a level root with contains the SI and DI service types represented as $S I$ and $D I$. Finally, these two groups contain the corresponding workflow instances. This results in three parameters $O F_{\text {root }}, O F_{S I}$ and $O F_{D I}$.

\subsection{Simulation approach}

The simulation environment contains a collection of start and stop events, that are queued. A timestamp is associated with each of the events, and they are processed in-order. When a stop event is processed, a new start event is generated denoting when the service is activated next. When a start event is processed, the workflow is added using the doPlace algorithm explained in 
Section 5, and based on the result of the algorithm execution the workflow is either added to the set of active services, the workflow is not started, or other workflows are aborted. Based on the resulting state, the required collection of start and stop events are generated. The events can then be analyzed to determine the percentage of workflows that were correctly executed, that were aborted, and that were not executed can be determined.

The SRNAID problem is used to determine whether services can be added to the system, and whether their activation could result in problems. To evaluate the algorithm, we require a difficult problem model, where enough services are active, and where there is little remaining network capacity, as otherwise there would never be a conflict: activating a collection of low-bandwidth services in a high-bandwidth network will never be a problem. Because of this, we use the SRNAID model to determine a minimally dimensioned network. We achieve this by, rather than maximizing the achieved $z_{w}$ values, instead minimizing the total network resources while every $z_{w}$ is assigned value 1 .

The resulting network resource configuration is the network with the smallest network capacities that, for the given services, still allows all of them to run. Using this hardest network, we can then simulate the use of the services for an extended period of time to determine the amount of violations, which can be used to determine the number of service workflows that fail. This approach can be used to determine the worst-case failures: any possible network that is accepted must have at least the same amount of capacity. If the network has higher capacity, the services will still be accepted, and failure rates will decrease.

\section{Evaluation}

\subsection{Quality}

\subsubsection{Medical communication use case}

We first evaluate the quality of the SRNAID algorithm by simulating the execution of a collection of workflows, and measuring the number of resource conflicts that occur. As mentioned, the networks are dimensioned using a modified SRNAID execution where network capacities are minimized, resulting in the tightest network where the regular SRNAID algorithm can still accept all workflows. In the simulation, four parameters can be changed: $O F_{\text {root }}, O F_{N C}$, $O F_{V o i P}$, and $O F_{\text {video. }}$. These values correspond to the overprovisioning factors for the root of the hierarchy and each of the three services. We also compare our results with a simpler flat, non-hierarchical approach, where only a single $O F$ is used. For every parameter configuration, three weeks of execution were simulated based on the hospital layout described in the previous section.

In Figure 7, the failure rates are shown in a scenario where all $O F$ factors are equal. We refer to this scenario as EqualOF. When this $O F$ factor is zero, all resources are shared, implying every network edge only has enough capacity to provide for a single video workflow (as video requires most bandwidth). As can be observed, the failure rate for video flows is extremely high, and there is also a high failure rate of VoIP calls. This is to be expected: as there is only 


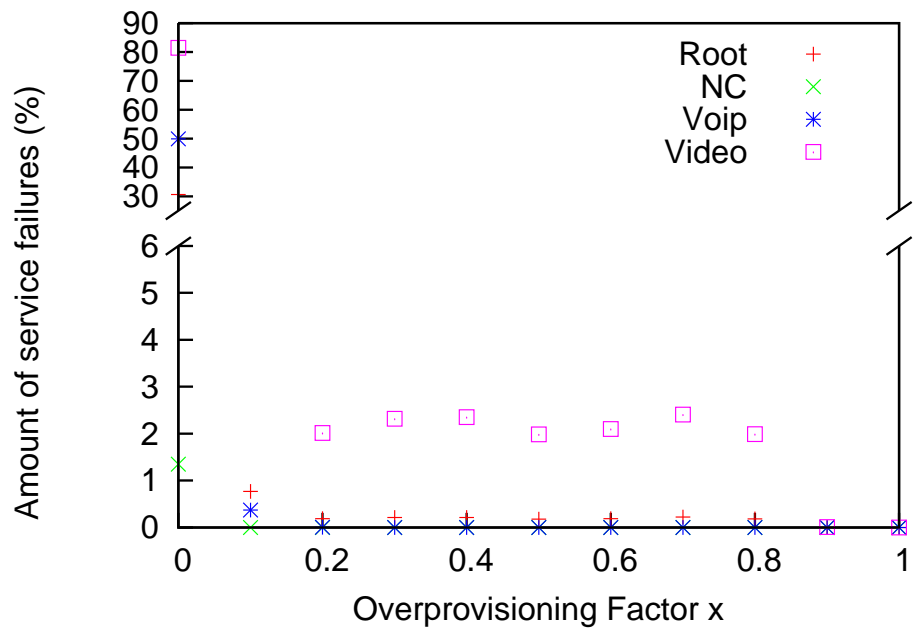

Figure 7: EqualOF: Fail rates using SRNAID in groups with varying overprovisioning factors. $O F_{\text {root }}=O F_{N C}=O F_{V o i P}=O F_{\text {video }}=x$

bandwidth for running a single video flow, starting any other workflow will cause the video flow to fail. Despite this very low capacity, there are still relatively few failures for the critical $\mathrm{NC}$ service due to its lower network demand and as the conflict management ensures it is preferred. As the common $O F$ factor is increased, the number of failures decreases. Once $O F \geq 0.2$, all $\mathrm{NC}$ calls succeed, but VoIP failures only stop once $O F \geq 0.9$, and at this point there are still some rare video flow failures.

Another choice of $O F$ values is the FixedRoot approach. This approach is similar to EqualOF, but the root factor $O F_{\text {root }}$ is assigned value 1. Intuitively, we can explain the FixedRoot approach as follows: we share resources between services of every service type, to ensure less network resources are needed, but assign a fixed amount of resources to the services themselves to ensure they minimally interfere with each other. As can be seen in Figure 8 the fail rates are noticeably lower: once the $O F$ values of the three services exceeds 0.3 no more failures occur in any service.

Finally, we consider a last approach, Flat, which does not make use of service hierarchies and considers all services equally, making use of only a single overprovisioning factor $O F$. The fail rates of this approach are shown in Figure 9, and are similar to those of EqualOF.

The required network capacity for the three approaches is shown in Figure 10. We observe that for the same choice of $O F$ a similar cost is achieved, with the EqualOF approach requiring the lowest amount of capacity, while the FixedRoot approach requires most resources. This is to be expected as the EqualOF shares resources twice, once for level in the hierarchy, while the FixedRoot and Flat approaches only share resources once. As the $O F_{\text {root }}=1$ 


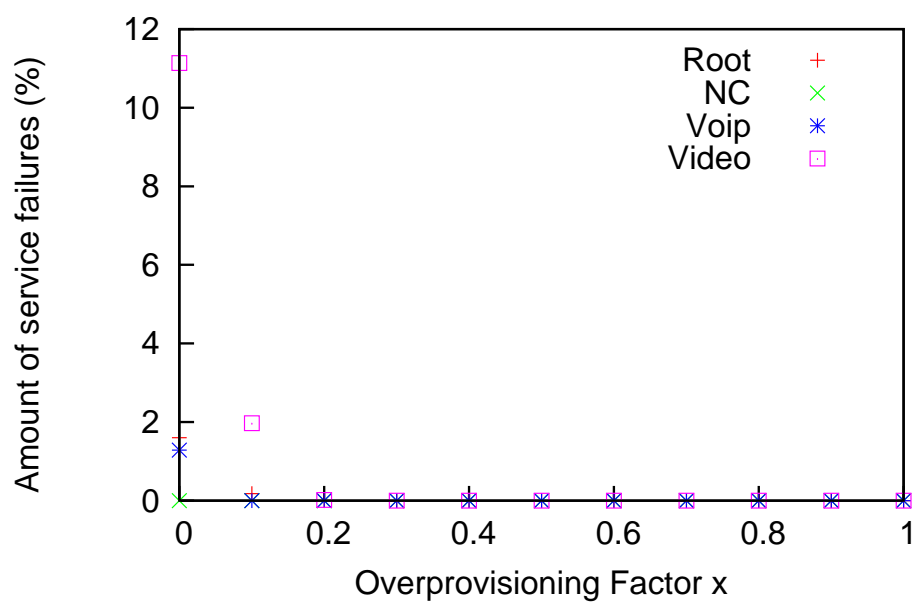

Figure 8: FixedRoot: Fail rates using SRNAID in groups with varying overprovisioning factors and fixed root overprovisioning. $O F_{\text {root }}=1, O F_{N C}=O F_{V o i P}=O F_{\text {video }}=x$

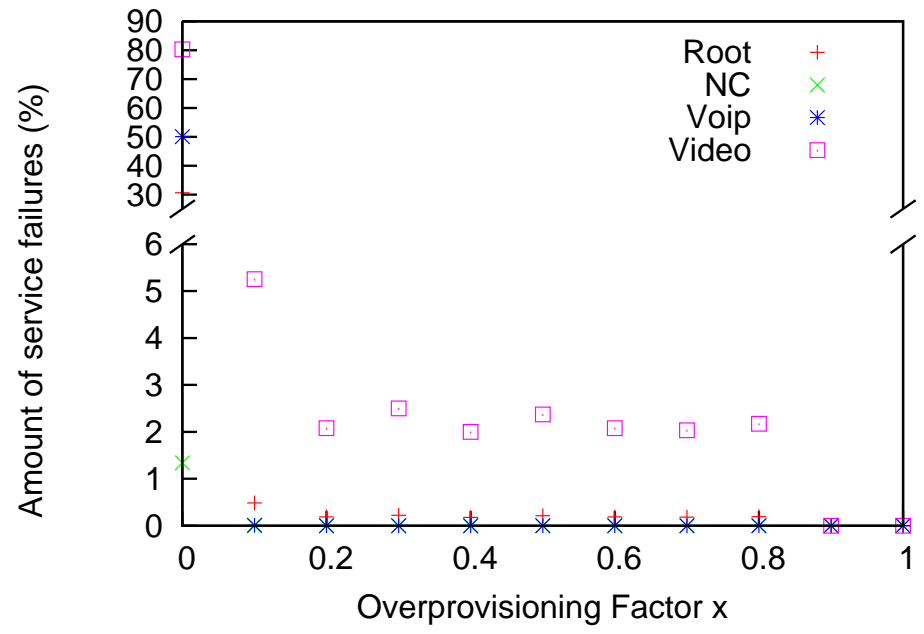

Figure 9: Flat: Fail rates using SRNAID without hierarchies. $O F=x$ 


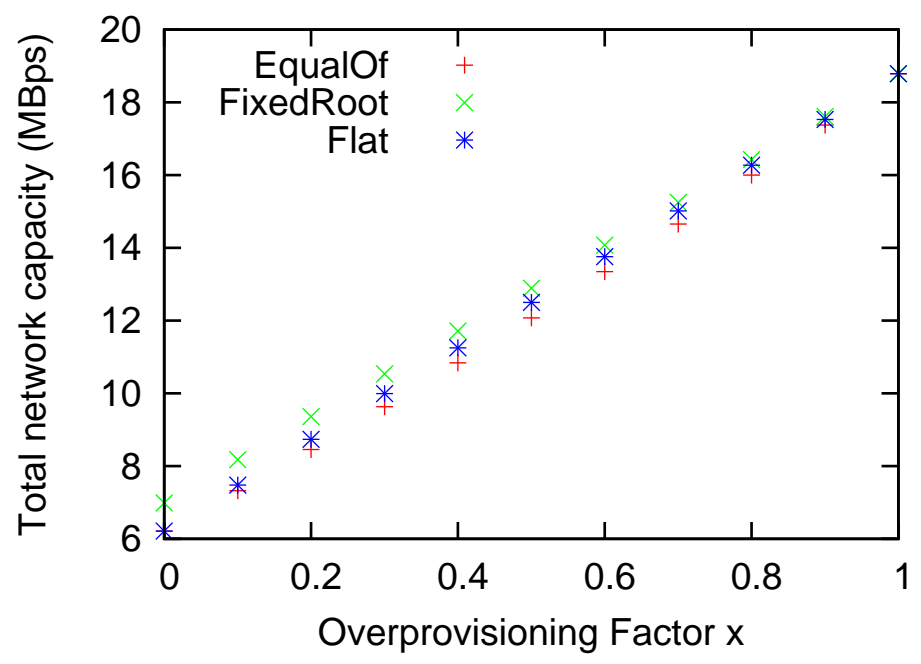

Figure 10: Required bandwidth for the three discussed SRNAID approaches with varying overprovisioning factors.

in the FixedRoot approach, there is slightly less resource sharing compared to the Flat approach, which is why it requires more resources. The case where $O F_{\text {root }}=O F_{N C}=O F_{V o I P}=O F_{\text {video }}=1$ corresponds to the regular NAID approach without resource sharing; in this case there can be no failure of workflows.

When combined with the failure rates, we can conclude that the hierarchical approach using fixed $O F_{\text {root }}$, FixedRoot, performs best, requiring only between 10 and $12 \mathrm{MBps}$ of network capacity to function without any failures, while the Flat and EqualOF approaches both require 18MBps of network capacity to achieve similar results. The latter network capacities are only slightly less than the required network capacity for the algorithm without resource sharing, which is 19MBps. For the considered cases, the FixedRoot approach requires $42 \%$ less resources than an approach without resource sharing, while still achieving similar qualitative results. The FixedRoot approach also requires $38 \%$ less resources compared to the Flat and EqualOF approaches to achieve similar results.

Using hierarchies to increase control of the resource sharing can thus greatly increase the number of services that can be allowed on a system, while at the same time also increasing the quality these services can achieve: the FixedRoot approach both needs less bandwidth and achieves better quality results than the Flat approach where no service hierarchies are used. The choice of parameters in the hierarchy is important, as evidenced by the worse results of the EqualOF approach. 


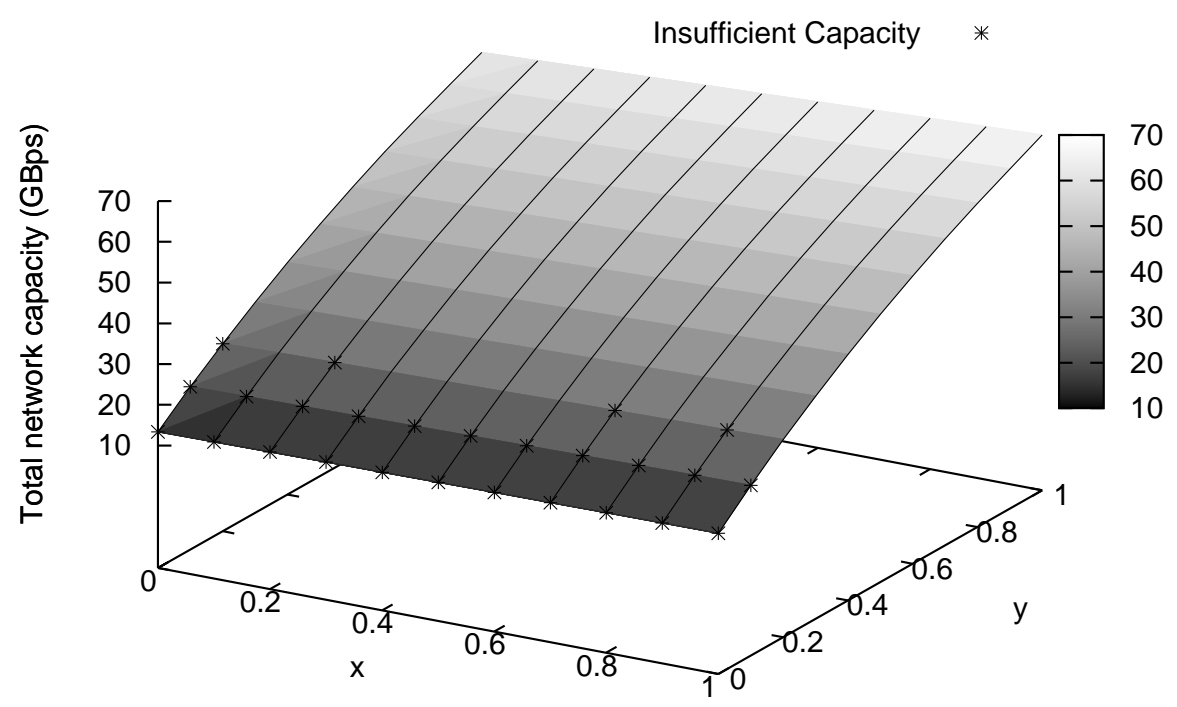

Figure 11: The required total network bandwidth for varying $O F$ parameters in the generated scenario $\left(O F_{\text {root }}=x, O F_{S I}=O F_{D I}=y\right)$. Markings show where workflow failures occurred during any of the simulation runs (DI, SI or balanced) due to insufficient network capacity.

\subsubsection{Generated use case}

We evaluate the SRNAID algorithm using the generated use case for various service frequencies $\alpha_{D I}$ and $\alpha_{S I}$ to determine the effect of the distribution of server and data intensive workflows. We consider three scenarios: (1) a Server Intensive (SI) scenario $\left(\alpha_{S I}=9, \alpha_{D I}=1\right),(2)$ a Data Intensive (DI) scenario $\left(\alpha_{S I}=1, \alpha_{D I}=9\right)$, and (3) a balanced scenario $\left(\alpha_{S I}=5, \alpha_{D I}=5\right)$. In the first scenario, SI workflows activate on average 9 times every time interval while the DI workflows only activate once every time interval. In the second scenario, the opposite happens while in the final scenario both workflow types activate on average 5 times during every time interval.

In Figure 11, the total network cost for various $O F_{\text {root }}, O F_{D I}$ and $O F_{S I}$ combinations is shown. The markings show the $O F$ values where any of the workflows failed during the simulation for any of the three scenarios (DI, SI or balanced). We observe that increasing $O F_{\text {root }}$ has a limited impact on both cost and quality compared to increasing $O F_{D I}$ and $O F_{S I}$. Using $O F_{D I}=O F_{S I}=$ 0.3 no failures occur in any of the simulations, irrespective of the chosen $O F_{\text {root }}$. With $O F_{D I}=O F_{S I}=0.2$ failures still occur sporadically, but only for DI workflows in the DI scenario (with a failure rate of less than 1\%). For lower $O F$ values, failures occur frequently for DI workflows in all scenarios. SI workflows only fail during simulation in the cases where $O F_{D I}=O F_{S I}=0$.

Figure 12 compares the workflow failure percentages for the three scenarios for a heavily underprovisioned network with $O F_{D I}=O F_{S I}=0$ where failures of every workflow type occur. We observe that the SI workflows generally have 


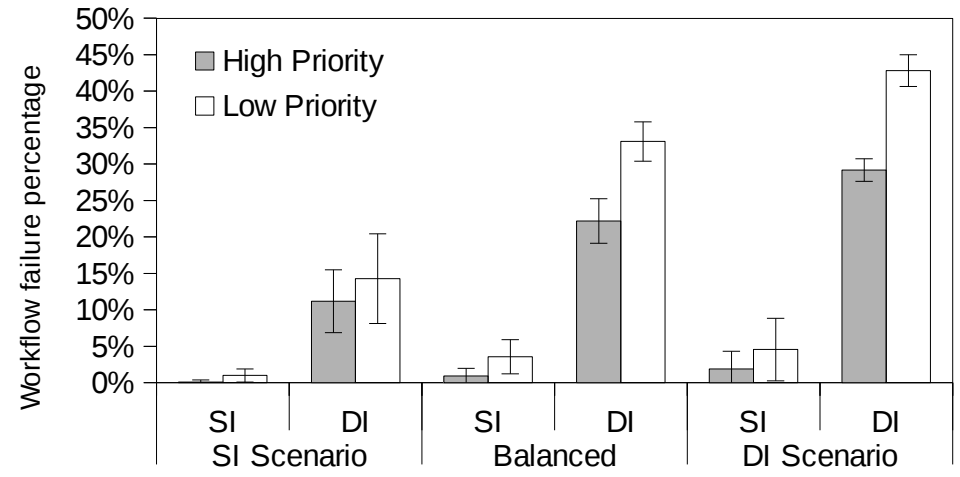

Figure 12: Failure rates of DI workflows in the DI, SI and balanced scenarios using a heavily underprovisioned network $\left(O F_{D I}=O F_{S I}=0\right)$.

lower failure rates compared to DI workflows, and that high priority workflows fail less frequently than low priority workflows. The SI scenario results in the lowest failure rate for all workflow types, while the DI scenario results in the highest number of failures. As was shown in Figure 11, increasing the $O F$ values quickly reduces the failure rates until no failures occur during simulation.

Without resource sharing, 67GBps network capacity is needed for provisioning all workflows. Using resource sharing, a significant reduction in resources can be achieved resulting in a total network demand of 32GBps without any failures during simulation, resulting in a capacity reduction of $\pm 52 \%$ compared to an approach without resource sharing. If infrequent workflow failure is tolerated, the total network capacity can be further reduced to $26 \mathrm{GBps}$ resulting in a capacity reduction of $\pm 61 \%$ compared to an approach without resource sharing.

This evaluation shows that the model can incorporate both network and server resource demand. It is however clear that network constraints are more stringent and result in more failures than server resource constraints. This is not surprising, as when there is sufficient capacity for most DI flows, there will almost always be sufficient residual network resources for the lower network demand required for the SI flows, even if the services used in the workflow are allocated further apart. This implies that, as long as there is a service where the service may run, wherever it is located within the network, there is likely to be sufficient network capacity between them for these workflows.

\subsection{Execution speed}

The execution speed of the SRNAID algorithm was evaluated using a server with dual-socket quad-core Intel Xeon L5420 processor and 16GB RAM. The base evaluation setup is the same as the that of the MC use case, but we incrementally increase the number of leaf nodes, resulting in an increase of active workflows. We make use of the FixedRoot approach as this yields the highest quality at the lowest resource requirement. The $O F$ factors for the SRNAID 


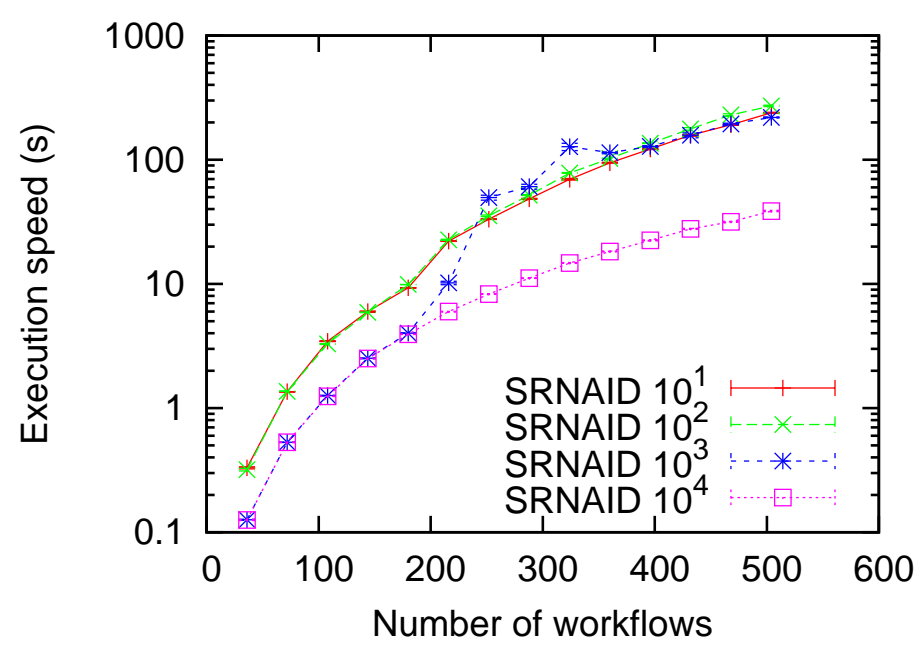

Figure 13: The execution speed of the SRNAID algorithm for varying network edge capacities. The SRNAID parameters are chosen based on the results of the quality evaluation: $O F_{\text {root }}=$ 1 , and $O F_{N C}=O F_{V o I P}=O F_{\text {video }}=0.3 .40$ iterations per data point.

algorithm are thus chosen as follows: $O F_{\text {root }}=1$, and $O F_{N C}=O F_{V o I P}=$ $O F_{\text {video }}=0.3$.

The results of this evaluation are shown in Figure 13, where the performance of the SRNAID algorithm is shown for increasing numbers of workflows. The SRNAID algorithm is executed four times, for input problems with varying edge capacities in $\left\{10^{1}, 10^{2}, 10^{3}, 10^{4} \mathrm{KBps}\right\}$. These varying capacities can impact the problem complexity, as it is easier to find a good solution if much network capacity is present. We incrementally increase the number of terminals per floor, which results in an increase in the number of possible workflows.

It can be seen in the Figure that the execution time of the algorithm increases as the number of workflows increases. The algorithms with limited network capacity $\left(10^{1}\right.$ and $\left.10^{2} \mathrm{KBps}\right)$ require more time to run than the algorithms with higher network bandwidths $\left(10^{4} \mathrm{KBps}\right)$ as it is more difficult determine a result that respects all of the constraints. This is especially noticeable for the scenario where there is $10^{3} \mathrm{KBps}$ network capacity is available: for smaller numbers of workflows, there is sufficient capacity making it perform similar to the case where there is $10^{4} \mathrm{KBps}$ of network capacity per edge. As the demand increases due to the increasing number of workflows, network capacity becomes a bottleneck, causing it to then start behaving like the problems with less available bandwidth.

When 500 workflows are active, the evaluations with more available bandwidth require only $16 \%$ of the execution time of the lower bandwidth algorithms. This difference can be explained, as when more bandwidth is available, it is much easier for the ILP solver to find a solution where every workflow can be 
completely executed. When there is insufficient bandwidth, it becomes more difficult to find the optimal solution, maximally satisfying the amount of allocated capacity. This is an interesting property, as when the SRNAID algorithm is used as an admission filter, it will mainly be used to validate the configuration: there will usually be sufficient capacity to execute all workflows, resulting in more favorable execution speeds. The worst-case performance will only occur in highly bandwidth constrained scenarios.

\subsection{Algorithm scalability}

As observed from Figure 13, the performance of the SRNAID algorithm is best when there is sufficient network and server capacity for all of the service workflows, making the impact analysis run faster. This has interesting consequences: if a workflow is deployed in a scenario where there is sufficient capacity, the SRNAID algorithm will finish quicker resulting in limited overhead. If there is less capacity, the algorithm will need more time to run. Thus, the SRNAID results in limited overhead if there is sufficient capacity, and only requires more execution time when it is actually important to run it to ensure there will be no service failures.

It is also important to note that the impact analysis is a step that is executed when new services are instantiated, a process which currently takes multiple days, making the process less sensitive to time constraints. A final consideration is that applying network impact analysis is more important for smaller client deployments, where the quality of the networks tends to be lower. In these cases, the number of workflows is usually limited to \pm 100 workflows. These factors make the long algorithm execution duration not prohibitive for the algorithm's use.

Despite these considerations, it is desirable to improve the scalability of the SRNAID algorithm to make it possible to obtain faster feedback. To improve the scalability of the approach when larger networks are used, it is possible to segment the input network, decomposing the problem network in separate networks, and statically dividing overlapping components between the networks. For the evaluation scenario, this can be easily done by considering both buildings within the simulation separately, leaving only the cloud uplink as a shared edge between both subproblems. Due to the symmetry in the problem model, the capacity of this shared edge can be divided equally between both subproblems. We will refer to this algorithm as the SR SPLIT algorithm. Two variants of the SR SPLIT algorithm can be discerned: SR SPLIT ${ }_{\text {seq }}$, where the SRNAID algorithm is invoked sequentially on the subproblems, and the SR SPLIT algorithm where both algorithms are executed in parallel on separate computation nodes. This approach is generally applicable to all input networks, but simulations may be needed to determine an optimal division of shared edges between subproblems if the input network is not symmetrical.

Figure 14 compares the execution speed of the SRNAID algorithm with that of SR SPLIT seq $_{\text {and SR SPLIT }}$.r. For this scenario, both SR SPLIT algorithms execute faster than the SRNAID algorithm, with the SR SPLIT par algorithm 


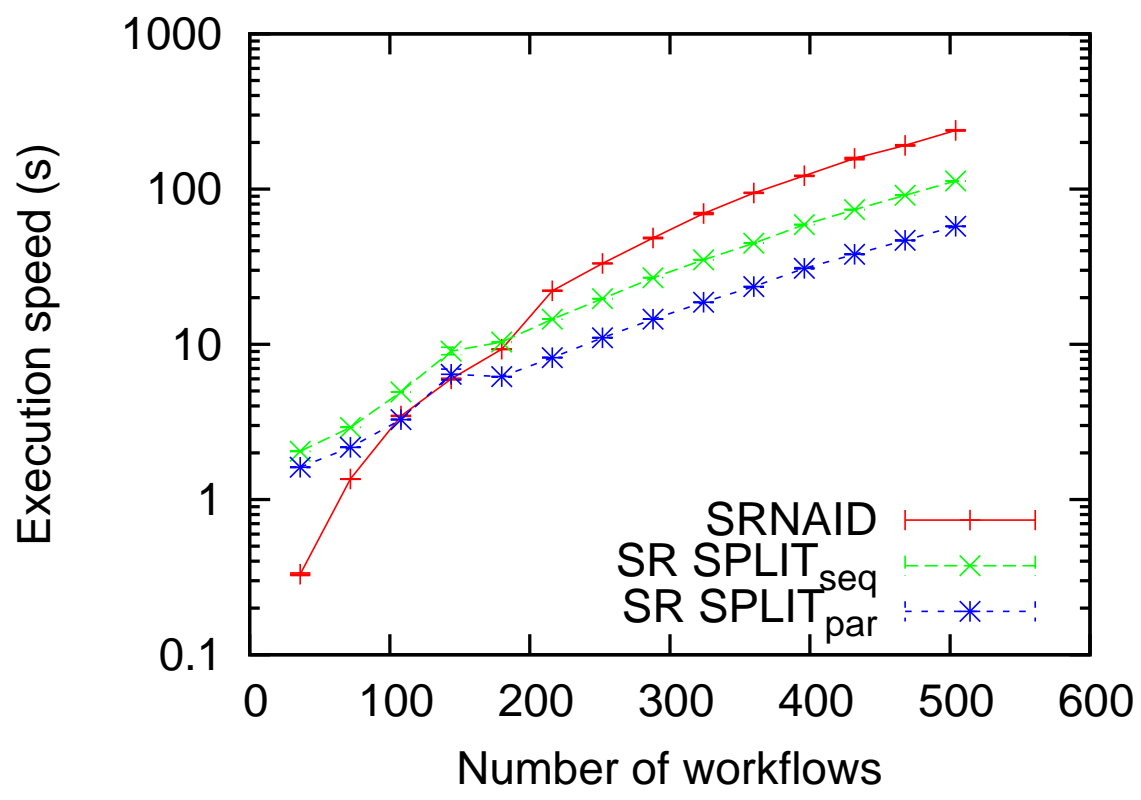

Figure 14: The execution speed of the SRNAID algorithm compared to the execution speed of the SR SPLIT algorithm. The algorithm parameters are chosen based on the results of the quality evaluation: $O F_{\text {root }}=1$, and $O F_{N C}=O F_{V o I P}=O F_{\text {video }}=0.3$. The edge capacity throughout the network is heavily constrainted (limited to 10KBps per edge) to illustrate worst case performance. 40 iterations for each data point.

needing about half the time to run compared to the SR SPLIT seq $_{\text {algorithm. }}$ The latter is to be expected as the network was split into two parts.

The impact on the total network requirement, calculated as discussed in Section 7.1 is negligible for this scenario: only $0.035 \%$ more network capacity is needed. The impact on the shared edge itself is however larger: there, a capacity increase of $4.6 \%$ is observed, which implies that for these edges there will need to be $4.6 \%$ overprovisioning compared to the SRNAID approach. Splitting the network into more subnets further increases parallelism and reduces the subproblem size, and is expected to further increase the execution speed if needed. As in such a scenario there are more shared edges, the amount of resources needed by the algorithm to accept a service configuration will however further increase.

\section{Conclusions}

In this article, we discussed how resource sharing can be incorporated when determining impacts of service workflows on a network during service deployment by defining a model that allows service workflows to partially ignore other workflows. By hierarchically specifying groups of workflows, fine-grained control over the resource sharing can be achieved, increasing the quality of resulting 
configurations. We also specified a runtime conflict management algorithm that can be used to resolve resource conflicts when resource conflicts occur during runtime. By assigning a priority to different workflows, the successful execution of important workflows can be ensured. We found that, when suitable hierarchy parameters are determined through simulation, the presented hierarchical SRNAID algorithm requires $\pm 42 \%$ and $\pm 52 \%$ less resources than an approach without resource sharing for two evaluation use cases, without any workflow failures occurring during runtime. We also discussed how the scalability of the algorithm may be improved.

We focused on the application of the SRNAID algorithm as an admission filter, where it is used to determine whether services with high-availability requirements can be provided on a given network. In the future, the SRNAID approach could also be used for a what-if analysis, determining which additional hardware and network resources are required for providing a service, making it easier accurately predict the cost of implementing additional or new services. In future work, the presented model can also be adapted for resource allocation, determining which servers and devices are responsible for which services.

\section{Acknowledgement}

Hendrik Moens is funded by the Institute for the Promotion of Innovation by Science and Technology in Flanders (IWT). This work was carried out using the Stevin Supercomputer Infrastructure at Ghent University, funded by Ghent University, the Hercules Foundation and the Flemish Government - department EWI. We thank Televic Healthcare for their input on our evaluation use case.

[1] Amazon, Amazon EC2 instance types (2014). URL http://aws.amazon.com/ec2/instance-types/

[2] H. Moens, E. Truyen, S. Walraven, W. Joosen, B. Dhoedt, F. D. Turck, Network-Aware Impact Determination Algorithms for Service Workflow Deployment in Hybrid Clouds, in: Proceedings of the 8th International Conference on Network and Service Management (CNSM 2012), 2012, pp. $28-36$.

[3] R. K. Ahuja, T. L. Magnanti, J. B. Orlin, Network flows, Prentice Hall, New Jersey, 1993. doi:10.1002/net.3230160411.

[4] B. Awerbuch, T. Leighton, Improved approximation algorithms for the multi-commodity flow problem and local competitive routing in dynamic networks, in: Proceedings of the 26th Symposium on Theory of Computing STOC, ACM, 1994, pp. 487-496.

[5] M. Pióro, D. Medhi, Routing, Flow, and Capacity Design in Communication and Computer Networks, Morgan Kaufmann Publishers Inc., San Francisco, CA, USA, 2004. 
[6] V. Kolar, N. B. Abu-Ghazaleh, A Multi-Commodity Flow Approach for Globally Aware Routing in Multi-Hop Wireless Networks, Proceedings of the 4th Annual IEEE International Conference on Pervasive Computing and Communications PERCOM06 (2006) 308-317doi:10.1109/PERCOM. 2006.3.

[7] W. Szeto, Y. Iraqi, R. Boutaba, A multi-commodity flow based approach to virtual network resource allocation, Proceedings of the IEEE Global Telecommunications Conference GLOBECOM 036 (2003) 3004-3008. doi : 10.1109/GLOCOM. 2003.1258787.

[8] Y. L. Y. Liu, D. Tipper, P. Siripongwutikorn, Approximating optimal spare capacity allocation by successive survivable routing (2005). doi:10.1109/ TNET. 2004.842220 .

[9] L. Zhao, Y. Ren, M. Li, K. Sakurai, Flexible service selection with userspecific QoS support in service-oriented architecture, Journal of Network and Computer Applications 35 (3) (2012) 962-973. doi:10.1016/j.jnca. 2011.03 .013$.

[10] J. Rolia, A. Andrzejak, M. Arlitt, Automating enterprise application placement in resource utilities, in: Self-Managing Distributed Systems: 14th IFIP/IEEE International Workshop on Distributed Systems: Operations and Management (DSOM 2003), Springer, 2004, pp. 118-129. doi:10.1007/978-3-540-39671-0\_11.

[11] C. Tang, M. Steinder, M. Spreitzer, G. Pacifici, A scalable application placement controller for enterprise data centers, in: Proceedings of the 16th international conference on World Wide Web, 2007, pp. 331-340. doi : $10.1145 / 1242572.1242618$.

[12] C. Adam, R. Stadler, Service Middleware for Self-Managing Large-Scale Systems, IEEE Transactions on Network and Service Management 4 (3) (2007) 50-64. doi:10.1109/TNSM. 2007.021103.

[13] F. Wuhib, R. Stadler, M. Spreitzer, Gossip-based Resource Management for Cloud Environments, in: Proceedings of the 6th International Conference on Network and Service Management (CNSM 2010), 2010, pp. 1-8.

[14] H. Moens, E. Truyen, S. Walraven, W. Joosen, B. Dhoedt, F. De Turck, Cost-Effective Feature Placement of Customizable Multi-Tenant Applications in the Cloud, Journal of Network and Systems Management 22 (4) (2014) 517-558. doi:10.1007/s10922-013-9265-5.

[15] H. Moens, J. Famaey, S. Latré, B. Dhoedt, F. De Turck, Design and Evaluation of a Hierarchical Application Placement Algorithm in Large Scale Clouds, in: Proceedings of the 12th IFIP/IEEE International Symposium on Integrated Network Management (IM 2011), 2011, pp. 137-144. doi:10.1109/INM. 2011.5990684. 
[16] C. Low, Decentralised Application Placement, Future Generation Computer Systems 21 (2) (2005) 281-290. doi:10.1016/j.future.2003.10. 003.

[17] D. Carrera, M. Steinder, I. Whalley, J. Torres, E. Ayguadé, Utility-based placement of dynamic web applications with fairness goals, in: Proceedings of the 11th Network Operations and Management Symposium (NOMS 2008), IEEE, 2008, pp. 9-16. doi:10.1109/NOMS.2008.4575111.

[18] J. Bronsted, K. M. Hansen, M. Ingstrup, Service Composition Issues in Pervasive Computing, IEEE Pervasive Computing 9 (1) (2010) 62-70. doi: 10.1109/MPRV.2010.11.

[19] K.-T. Tran, N. Agoulmine, Y. Iraqi, Cost-effective complex service mapping in cloud infrastructures, in: Proceedings of the 13th Network Operations and Management Symposium (NOMS 2012), 2012, pp. 1-8.

[20] C. Develder, J. Buysse, M. De Leenheer, B. Jaumard, B. Dhoedt, Resilient network dimensioning for optical grid/clouds using relocation, in: 2012 IEEE International Conference on Communications (ICC), 2012, pp. 62626267. doi:10.1109/ICC.2012.6364981.

[21] B. Sansó, M. Gendreau, F. Soumis, An algorithm for network dimensioning under reliability considerations, Annals of Operations Research 36 (1) (1992) 263-274.

[22] S. Verma, R. K. Pankaj, A. Leon-Garcia, Call admission and resource reservation for guaranteed quality of service (GQOS) services in internet, Computer Communications 21 (4) (1998) 362-374. doi:10.1016/ S0140-3664 (97)00169-2.

[23] S. Sharafeddine, Capacity assignment in multiservice packet networks with soft maximum waiting time guarantees, Journal of Network and Computer Applications 34 (1) (2011) 62-72. doi:10.1016/j.jnca.2010.09.004.

[24] R. Carter, M. Crovella, Measuring bottleneck link speed in packet-switched networks, Performance evaluation 27 (28) (1996) 297-318.

[25] N. Hu, P. Steenkiste, Evaluation and Characterization of Available Bandwidth Probing Techniques, JSAC 21 (6) (2003) 879-894.

[26] M. Li, Y.-L. Wu, C.-R. Chang, Available bandwidth estimation for the network paths with multiple tight links and bursty traffic, Journal of Network and Computer Applications 36 (1) (2013) 353-367. doi:10.1016/j.jnca. 2012.05 .007$.

[27] Scala 2.9.2 (2014). URL http: //www.scala-lang.org/ 
[28] IBM ILOG CPLEX 12.4 (2014).

URL http://www-01.ibm.com/software/integration/optimization/ cplex-optimizer 